\title{
Presente y futuro de las relaciones comerciales de Canadá y Estados Unidos frente a los nuevos desafíos
}

\author{
Delia Montero C.* \\ EnRIQue Pino Hidalgo**
}

\section{RESUMEN}

En este artículo se analiza el impacto comercial de China en el comercio entre Canadá y Estados Unidos. A pesar de que Canadá perdió su posición histórica como primer socio comercial de Estados Unidos, debido al ascenso de las exportaciones de China al mercado estadunidense, esto no ha afectado la estructura de sus intercambios comerciales. Las exportaciones chinas a Estados Unidos son principalmente manufacturas intensivas en mano de obra y capital, en tanto que las exportaciones canadienses son principalmente de recursos naturales y servicios. Además, ambos países tienen acuerdos de defensa y seguridad que agilizan el comercio y permiten a Estados Unidos enfrentar mejor las amenazas externas.

Palabras clave: comercio, competitividad, TLCAN, recursos naturales, entorno institucional

\section{ABSTRACT}

The authors analyze China's impact on U.S.-Canadian trade. Despite Canada's having lost its historic position as the United States' first trade partner due to the hike in Chinese exports to the U.S. market, this has not affected the structure of their commercial operations. Chinese exports to the U.S. are mainly labor- and capital-intensive manufactured goods, while Canadian exports are mainly natural resources and services. In addition, the two countries have defense and security accords that facilitate trade and allow the United States to deal better with external threats.

Key words: trade, competitiveness, NAFTA, natural resources, institutional environment

\footnotetext{
* Profesora investigadora del Departamento de Economía, UAm Iztapalapa, <del@xanum.mx>.

** Profesor investigador adscrito al Departamento de Economía, UAM Iztapalapa, <pihen@prodigy.net.mx>. Se agradece a Jorge Hernández G. su colaboración para este texto.
} 
Las relaciones económicas, políticas y culturales entre Canadá y Estados Unidos se remontan a las etapas fundacionales de las colonias inglesas y francesas en Norteamérica a lo largo de los siglos XVII y XVIII, asociadas al fenómeno geoeconómico de compartir la frontera más grande del mundo. Este factor ha influido decisivamente en el establecimiento de una relación multidimensional sólida, única y vital en diversos ámbitos como el comercio, las inversiones, el tema fronterizo y la seguridad nacional, así como en la seguridad energética, entre otros.

El entorno institucional económico, político y cultural de Canadá ha sido una de las claves que explican el intenso y diverso flujo de inversiones y comercio que mantienen ambos países. Indudablemente, las corrientes de capital e inversiones fueron la consecuencia de una elaborada estructura de incentivos y ventajas mutuas muy poderosas dirigidas a propiciar la entrada de las firmas estadunidenses y el intercambio comercial, fortalecidos por una sólida definición de los derechos de propiedad y los contratos.

El llamado ambiente de negocios se reflejó en el rápido despliegue de la industria que emergió en Canadá en el siglo XIX, la cual, desde sus inicios, estuvo ampliamente vinculada con la expansión manufacturera, comercial y la pujanza financiera del noreste del territorio estadunidense. De ahí que la relación económica y política que han mantenido ambos países ha generado desde entonces una suerte de integración silenciosa.

A lo largo del siglo xx, la relación entre estas dos economías ha pasado por diversos acuerdos formales e informales que han definido importantes cambios en la dirección de una creciente integración económica, acuerdos políticos y estratégicos que han conducido a la firma del Tratado de Libre Comercio de América del Norte (TLCAN), el cual ha fungido como el punto de amarre de una serie de negociaciones comerciales bilaterales entre ambos países.

A pesar de los intentos de Canadá por diversificar y extender sus relaciones comerciales más allá del territorio estadunidense, observamos que los resultados son modestos y representan un proceso difícil si consideramos que más del 70 por ciento de su comercio continúa realizándolo con Estados Unidos. Si bien es cierto que con la firma del tLCAN México entró en el escenario canadiense, no fue sino hasta el 2001 cuando apareció entre los diez primeros países con los que tiene comercio Canadá. Al concluir 2011, México ocupaba el cuarto lugar como socio comercial de ese país, cuya economía importaba bienes por veinticinco mil millones de dólares que representaban el 5.5 por ciento del total de las importaciones canadienses; no obstante, cabe destacar que el intercambio comercial entre ambas naciones es, hasta cierto punto, marginal, ya que el comercio total representa menos del 5 por ciento del comercio total de Canadá. 
Durante la primera década del siglo xxI, se registraron cambios importantes en el comercio entre Estados Unidos y Canadá, atribuibles principalmente a la entrada de un nuevo actor en la escena internacional: China, cuyas exportaciones hacia aquellos dos países comenzaron a mostrar una tendencia ascendente y en pocos años se convirtió en el primer socio de Estados Unidos y en el segundo de Canadá y México. En esta dinámica, en 2011, las importaciones procedentes de China significaron el 18.4 por ciento del total de las importaciones estadunidenses, seguida por la Unión Europea (con el 16.6), Canadá (con el 14.4) y México, que aportó el 11.7 por ciento del total de las importaciones de Estados Unidos (wTо, 2011).

La tesis que planteamos en este trabajo enfatiza que a pesar de que Canadá perdió su posición histórica como primer socio comercial de Estados Unidos, debido al vertiginoso ascenso de las exportaciones de China al mercado estadunidense, consideramos que este cambio no ha afectado de forma determinante la estructura de los intercambios comerciales que realiza Canadá con Estados Unidos por varias razones. La primera se refiere a que las exportaciones chinas a Estados Unidos corresponden principalmente a productos de sectores manufactureros intensivos en mano de obra e intensivos en capital, en tanto que las exportaciones canadienses al vecino del Sur en buena medida son bienes basados en recursos naturales, petróleo y servicios que, como tales, no enfrentan la competencia china en los mercados de Estados Unidos.

La segunda razón es más específica y es que Canadá es uno de los principales proveedores de energéticos a Estados Unidos, en tanto que la tercera razón se relaciona con el carácter estratégico de Canadá que se expresa en la suscripción de los compromisos y pactos entre ambas naciones, que se concreta en acuerdos en materia de defensa y seguridad que permite hacer frente a posibles amenazas externas.

En la primera parte de este trabajo argumentamos cómo, a partir de los años sesenta, se formaliza y desarrolla el proceso de integración comercial de Canadá con Estados Unidos, que se confirma con la suscripción del estratégico acuerdo AutoPac (Canada-U.S. Automotive Products Trade Agreement o Acuerdo Comercial Automotriz Canadá-Estados Unidos). En la segunda parte, revisamos los cambios registrados en la estructura y el volumen del comercio de Canadá y Estados Unidos con China, así como sus impactos en las economías de los dos primeros países. Finalmente, realizamos un análisis para ver prospectivamente la dirección de las relaciones comerciales y financieras de Canadá y Estados Unidos, y los riesgos que enfrenta la economía del primero. 


\section{EL CAMINO HACIA LA INTEGRACIÓN COMERCIAL de Canadá y Estados Unidos}

Las relaciones comerciales entre estos dos países siempre han sido bastante estrechas; sin embargo, en la década de los setenta se registró un cambio fundamental, en particular en el trato al capital extranjero, principalmente estadunidense, que ya controlaba el 36 por ciento de todo el capital empleado en la industria de Canadá. Ciertamente, la penetración de la inversión extranjera había cobrado gran importancia en algunos sectores, en especial en el petrolero y el gas natural, en los que el capital foráneo representaba el 76 por ciento del capital invertido; en el sector minero, el control ascendía al 70 por ciento y en el manufacturero dominaba con el 61 por ciento del capital total invertido (Laliberté, 1992: 6).

La mayor presencia del capital extranjero en la economía canadiense en los años setenta fue precedida por la firma en 1965 del AutoPac, acuerdo diseñado para permitir a los productores norteamericanos (las corporaciones multinacionales estadunidenses ubicadas en Canadá, principalmente) racionalizar las plantas productoras mediante la remoción de las tarifas aduanales canadienses y estadunidenses sobre los embarques transfronterizos de vehículos terminados y autopartes originales. Específicamente, el AutoPac se abrió sobre las bases de la exención de impuestos de las exportaciones de los productos automotrices originarios de Canadá al mercado de Estados Unidos

Este acuerdo se formalizó y entró en operación antes de que Canadá iniciara una política basada en el ascenso del nacionalismo en los años setenta, con el gobierno de Pierre Elliot Trudeau, quien puso en marcha una nueva reglamentación para canadianizar la economía. Esta situación empezó a cambiar en 1974, con un replanteamiento de la política respecto de las inversiones extranjeras en sectores estratégicos, por lo que se crearon nuevas instituciones como la Foreign Investments Review Agency (FIRA), cuyo objetivo fue evaluar y regular las inversiones extranjeras, así como revisar las adquisiciones de activos existentes por parte de extranjeros.

Para comprender adecuadamente la evolución y los cambios experimentados por las relaciones comerciales y financieras entre Canadá y Estados Unidos, esbozamos una periodización que propone cuatro etapas en las que se que enfatizan los trazos principales de este proceso durante el último tercio del siglo xx. La primera corresponde a la década de los setenta, cuando el gobierno canadiense creó la FIRA, agencia encargada de regular las inversiones extranjeras bajo el gobierno liberal de Trudeau. Este organismo se encargó de reglamentar y vigilar tanto las inversiones que entraban como las que salían, según el criterio de que los flujos de capital fueran provechosos para el país. 
En 1980, de acuerdo con la estrategia nacionalista de Trudeau, también se creó el Programa Nacional de Energía que, entre otras cosas, monitoreaba las propiedades de los extranjeros en el sector de los energéticos. El resultado de esta política fue la disminución considerable del radio de control del capital foráneo, el cual pasó de 36 por ciento a finales de 1971, a 23 por ciento a finales de 1986; sin embargo, la década de los ochenta registró nuevamente cambios importantes, marcados por la apertura económica y la posterior firma y consolidación del Tratado de Libre Comercio de América del Norte (TLCAN).

La segunda etapa inició en 1985, con el reemplazo de la agencia FIRA por otra institución denominada Investment Canada, impulsada por el gobierno conservador de Brian Mulroney, quien llegó al poder en 1984 con una intensa política liberal en la aplicación de criterios sobre las inversiones foráneas, que estimaba se traduciría en una estructura de incentivos superior. En 1985, los objetivos del gobierno de Canadá se centraban en el fortalecimiento de la competencia y los temas de seguridad nacional. El gobierno liberal reafirmó el compromiso de reactivar la actividad comercial y promover la "defensa de Occidente", el control de las armas y el impulso de una política de desarme para reforzar la seguridad del país.

De manera específica, la política económica canadiense se centraba, de forma importante, en una serie de cambios estructurales que iniciaron a mediados de los ochenta, que comprendía un programa abierto de privatización de empresas del Estado, con el propósito de reemplazar el capital público por el privado. En la nueva estrategia liberal, también se incluyó la privatización y la desregulación del transporte, las comunicaciones y los servicios públicos. Acorde con la redefinición liberal del Estado, también fueron reducidos los servicios que proporcionaba el gobierno (Cameron, 2000: 221-228).

La tercera etapa inició con la firma del acuerdo comercial (FTA, por sus siglas en inglés) entre Estados Unidos y Canadá en 1989; aunque de corta duración, fue uno de los logros más importantes en la política de apertura económica, en la medida en que no sólo reafirmaba e intensificaba la relación comercial con Estados Unidos, sino que también fortalecía las inversiones entre ambas economías. En el marco de este acuerdo bilateral, la economía canadiense transitó de una integración de facto a una de jure con Estados Unidos, y de esta forma reafirmó la fortaleza de los vínculos económicos y financieros que tomó la relación bilateral entre estos dos países.

Finalmente, la cuarta etapa se consolidó con la firma del TLCAN. El nuevo acuerdo tripartito fue negociado según el principio de reciprocidad que incluía, en primer término, el establecimiento de relaciones preferenciales entre los países asociados. Al mismo tiempo, el tratado ofrecía una serie de incentivos a las tres economías asociadas en el marco de un espacio comercial sometido a reglas y mecanismos comunes. 
Consecuentemente, la puesta en marcha del TLCAN demandó el diseño y el establecimiento de un conjunto de instituciones y organismos que administraran y vigilaran la aplicación del nuevo entramado de reglas y normas que regularían el comercio, las inversiones y el ejercicio de los derechos de propiedad, etc., así como el cumplimiento de los compromisos asumidos y la instrumentación de los litigios. En este escenario, las emergentes reglamentaciones pactadas aseguraron el intercambio comercial con nuevas condiciones dirigidas a reducir los costos de transacción asociados con el comercio de bienes y servicios y los flujos de inversión entre los países signatarios. En este sentido, el TLCAN estableció una serie de acuerdos formales previos y otros no escritos, cuyas reglas se aplicarían en los tres países.

Para Estados Unidos, estaba claro que la firma de este acuerdo trilateral con sus socios vecinos de América del Norte profundizaría la relación comercial y financiera, al tiempo que permitiría mayores facilidades a la inversión extranjera. Para Canadá, el acuerdo formalizaba y perfeccionaba un largo proceso histórico de integración con Estados Unidos que permitía consolidar formalmente una relación de décadas, al generar una nueva estructura de facilidades de tipo arancelario, en transportación de bienes, etc., lo que posibilitó disminuir los costos de transacción de empresas, inversionistas, consumidores, etc., así como proteger los derechos de propiedad y, en general, aprovechar las nuevas ventajas y ganancias de la integración comercial en América del Norte.

En realidad, el TLCAN, en su carácter de acuerdo de integración comercial y financiera, vinculó a tres economías muy diferentes, con marcadas asimetrías, al tiempo que Canadá y México reafirmaron la fuerte gravitación respecto de Estados Unidos, principal mercado de sus exportaciones. En efecto, las asimetrías entre estas economías son notables y han tendido a acentuarse más que a disminuir como se planteó en el proyecto. Podemos revisar algunos indicadores. Por una parte, Canadá tiene una población de casi 34 millones de habitantes, un PIB per cápita de cincuenta mil dólares para 2012 y su inversión bruta en investigación y desarrollo fue del 2 por ciento del PIB. En tanto que México, cuyo proceso de integración comercial en el marco de la globalización es más reciente, ${ }^{1}$ tiene una población de 113 millones de habitantes, un PIB per cápita de diez mil dólares para el 2012, y su inversión bruta en investigación y desarrollo en 2000-2007 fue del 0.4 por ciento del PIB. Se trata, entonces, de relaciones claramente asimétricas, con efectos adversos en materia de competitividad, ingresos y beneficios comerciales, cuyo centro de gravitación se ubica en Estados Unidos.

\footnotetext{
${ }^{1}$ Algunos grupos industriales de Monterrey iniciaron negociaciones a partir de los setenta y establecieron filiales o hicieron algún tipo de alianza estratégica con empresas estadunidenses. Los casos más emblemáticos que registran este proceso corresponden a las corporaciones lfa, Cemex, Femsa, Gruma y Vittro (Correa y Villarreal, 2007: 131).
} 
Para Estados Unidos el TLCAN aseguraba la creación de un espacio comercial y financiero integrado con Canadá y México que ofrecía la posibilidad de reestructurar las actividades de las empresas filiales estadunidenses, así como la oportunidad de redimensionar la industria norteamericana en términos de competitividad (Deblock, 2012). Basta revisar la conformación y el funcionamiento del sector automotriz, dominado por tres empresas transnacionales estadunidenses: Ford, General Motors y Chrysler, que operan muy integrada y eficientemente en los tres países (Marcial, 2013). En el caso canadiense, se advierte cómo esta industria definida como una de las más importantes está dominada por las filiales estadunidenses. En efecto, de acuerdo con la Asociación Canadiense de la Industria Automotriz, esta rama genera empleo para aproximadamente quinientos mil canadienses en la producción de vehículos ligeros, camiones, autobuses y autopartes.

Con la puesta en marcha del TLCAN, el mercado de Estados Unidos se reafirmó como el principal destino de las exportaciones de Canadá y México. En efecto, cerca de las cuatro quintas partes de las exportaciones de Canadá y México gravitan alrededor de la economía estadunidense, mientras que el comercio entre Canadá y México, si bien ha experimentado un crecimiento relativamente rápido, en el intercambio arrancó desde un nivel muy bajo. En esta condición, las exportaciones de Canadá a México tienen un peso marginal, en tanto que las mexicanas a Canadá han cobrado cierta relevancia y representan el 4 o 5 por ciento del total exportado por México (Pino, 2013).

Como se observa en la gráfica 1, en 1995, casi el 80 por ciento del total de las exportaciones canadienses se dirigían a Estados Unidos, mientras que en ese mismo año, las importaciones provenientes de este país representaron el 66 por ciento, cifras que en 2000 constituyen el 86 por ciento y 64 por ciento, respectivamente. Esta tendencia se mantuvo hasta el 2007, pero en 2008 empiezan a descender y caen abruptamente tanto las importaciones como las exportaciones, debido a la crisis económica que azotó a Estados Unidos en ese año; no obstante, la gráfica indica una recuperación importante en 2011, hasta los niveles alcanzados en 2007, de tal forma que hacia 2011 la balanza comercial de Canadá con Estados Unidos nuevamente se restablece con un amplio superávit.

Cabe subrayar que de 1995 a 2008 la balanza comercial de Canadá con Estados Unidos ha sido en términos generales favorable; sin embargo, a pesar de la recuperación en 2011, la balanza comercial de Canadá no registra ya el mismo nivel del superávit que la caracterizaba, es decir, la economía canadiense pierde ventajas comerciales significativas con su principal socio. 


\section{Gráfica 1}

CANADÁ: COMERCIO CON ESTADOS UNIDOS Y EL MUNDO (1995-2011)

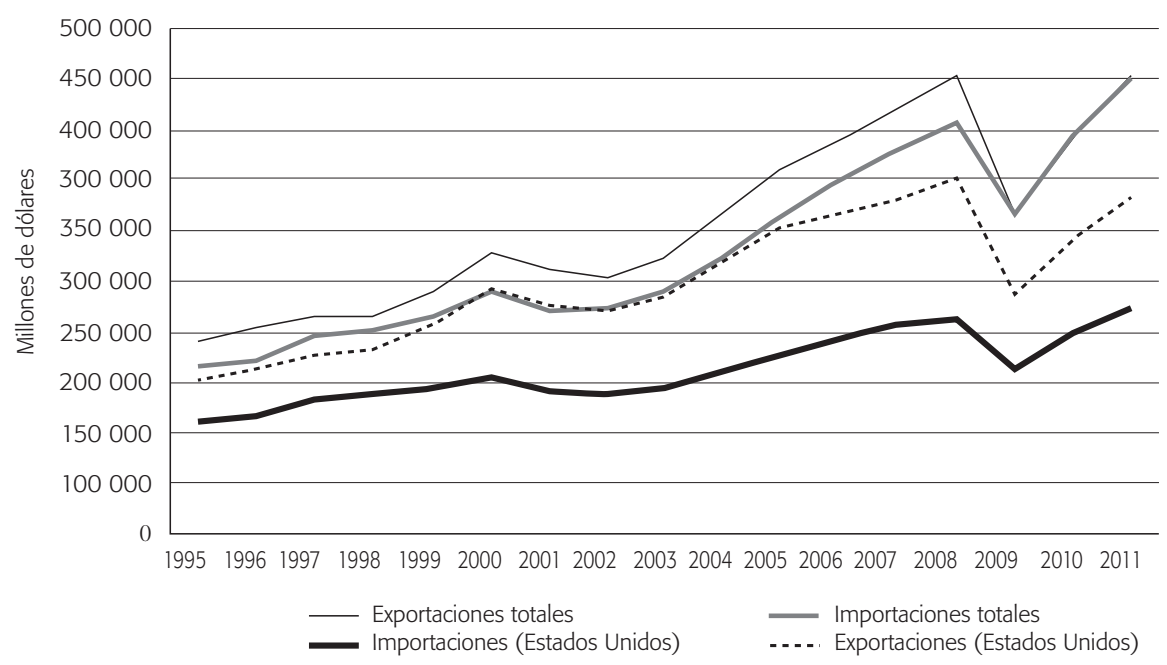

Fuente: Industry Canada, 2012.

En el caso de México, también vemos una fuerte concentración del comercio con Estados Unidos. Como se muestra en la gráfica 2, hacia el 2000, del total de las exportaciones mexicanas, el 88 por ciento se dirigía a Estados Unidos, mientras que para ese mismo año las importaciones provenientes de ese país eran del 73 por ciento respecto del total. En 2011, las exportaciones a Estados Unidos disminuyeron y representaron un 78 por ciento respecto del valor total de las exportaciones mexicanas. Los efectos de la crisis del 2008 también se reflejaron en las relaciones comerciales de México con Estados Unidos, pero a partir de 2009 se registró un significativo incremento anual en las exportaciones y en 2011, el saldo comercial para México fue muy favorable. Al igual que Canadá, la balanza comercial de México se ha mantenido superavitaria, incluso durante toda la primera década del nuevo siglo.

Como se observa en las gráficas 1 y 2, la expansión de los intercambios comerciales en el marco del TLCAN ha tenido efectos positivos para las dos economías que giran alrededor del mercado de Norteamérica, si consideramos que el comercio de Canadá y México con dicho mercado se ha incrementado notablemente, por lo que ambos países mantienen una balanza comercial bastante favorable. Ahora bien, los intercambios entre México y Canadá también se han incrementado rápidamente, aunque partieron de un nivel sumamente modesto. Las importaciones de Canadá provenientes de México pasaron de 399 millones de dólares en 1995, a 8120000000 de dólares en 2000 y a 24843000000 en 2011. Sin embargo, las exportaciones canadienses 


\section{Gráfica 2}

MÉXICO: COMERCIO TOTAL CON ESTADOS UNIDOS (2000-2011)

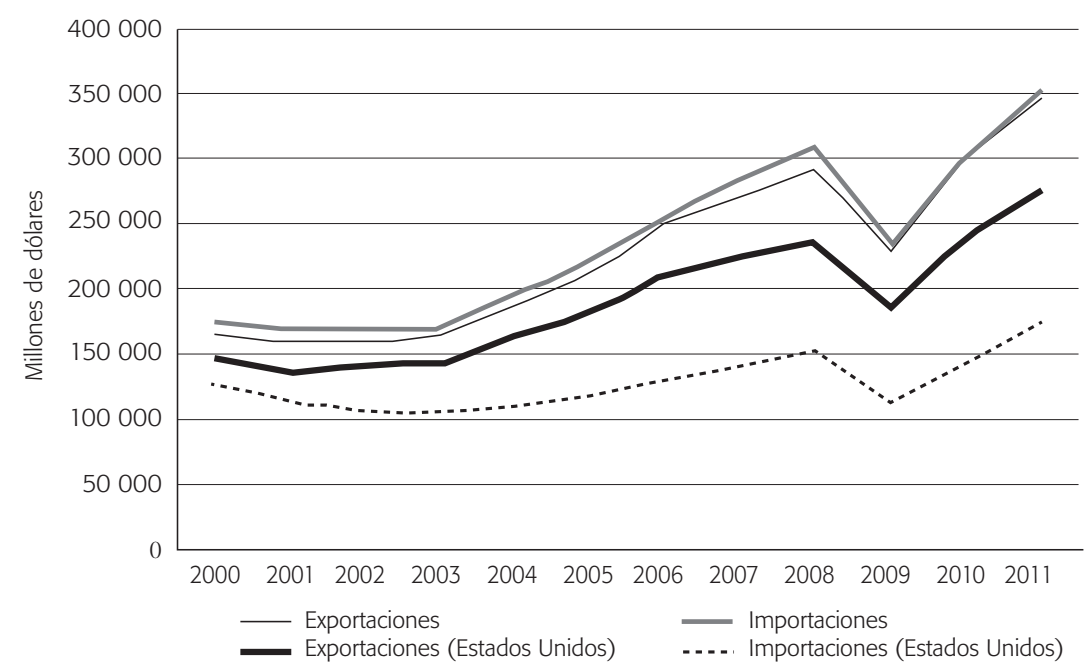

Fuente: Secretaría de Economía, 2012.

a México se mantienen en un nivel extremadamente bajo al representar sólo el 1.2 por ciento del total para el año mencionado, que lo coloca en el quinto lugar como mercado de los productos canadienses (wTO).

Diariamente, México y Canadá realizan intercambios de bienes por más de 52 millones de dólares que para la economía mexicana se traducen en un importante superávit, ya que nuestro país exporta a este país socio casi dos veces más que naciones como Reino Unido y tres veces más que Francia o Corea del Sur. Además, las exportaciones mexicanas a Canadá en 2009 sumaron 14619000000 de dólares, una cifra superior al valor de las exportaciones totales del resto de los países de América Latina hacia la economía canadiense.

Dicha economía tiene dos características que resultan ser muy atractivas para los intereses de Estados Unidos: la primera es que sus exportaciones descansan de manera importante en la explotación y el comercio de recursos naturales; la segunda, su fuerte vocación por la explotación y exportación de petróleo hacia el mercado estadunidense, que pone de relieve la gran concentración que presenta su estructura de exportaciones respecto de este bien estratégico y la alta gravitación que la economía tiene al respecto.

Canadá también es un socio estratégico en el complejo ámbito de la seguridad regional de Estados Unidos y como tal forma parte de los dispositivos de defensa, 
como es el cinturón de seguridad frente a posibles ataques. En efecto, los gobiernos de ambos países acordaron en 2002 la puesta en marcha del proyecto denominado "Fronteras inteligentes", consistente en una serie de normas que aseguran la eficiencia y la seguridad en el comercio entre ambas naciones y garanticen al mismo tiempo la seguridad nacional en sus territorios (Christie, 2002).

A pesar de sustentar oficialmente el multilateralismo comercial, en la práctica, en la nación canadiense se observa cómo las acciones de los diversos gobiernos se han orientado a mantener una relación preferencial con Estados Unidos, su socio privilegiado. Desde esta perspectiva, 2005 fue especialmente significativo, ya que las autoridades canadienses impulsaron una nueva revisión de su política exterior. En efecto, el primer ministro Paul Martin manifestó que una de las prioridades de Canadá era la estabilización de las relaciones bilaterales con Estados Unidos, al considerar categóricamente que este vínculo era una de las relaciones económicas más fuertes del mundo, a pesar de ser bastante asimétrica. ${ }^{2}$ Martin afirmó que dicha relación debía mantenerse fuerte, progresiva, fructífera y centrada en objetivos comunes, como la seguridad de las fronteras, la salud de la economía, además de garantizar la libre circulación de bienes y servicios entre ambos países.

El primer ministro Martin también reconoció que la redefinición del equilibrio del poder en el mundo había propiciado la aparición de nuevos países como fuerzas militares y económicas, y recordó que son muchas las potencias establecidas que luchan por mantener su influencia. Esta nueva revisión de la visión estratégica canadiense se ha sustentado en tres postulados fundamentales:

1. La seguridad de las fronteras ante las nuevas amenazas, principalmente el terrorismo. ${ }^{3}$

2. La necesidad de incrementar el comercio y la inversión, sobre todo con Estados Unidos y enseguida con Asia.

3. Fortalecer el multilateralismo (DFAIT, 2005).

\footnotetext{
${ }^{2}$ Las asimetrías se evidenciaban desde antes y se han ampliado. Por ejemplo, el monto de exportaciones de Canadá en 2011 alcanzó la cifra de 452000000000 de dólares y las importaciones sumaron 463000000000 , frente a las exportaciones e importaciones de Estados Unidos que sumaron 1480000000000 de dólares y 2265000000 000, respectivamente. El PIB canadiense en el mismo año representó 1736000000000 de dólares, una cifra ocho veces inferior al Рів de Estados Unidos que registró 14991000000000 de dólares. Entre 2009 y 2011, el comercio por habitante en Canadá representó una cifra de 28000 dólares, en tanto que para Estados Unidos significó 13000000000 de dólares. Por último, la población canadiense de 34000000 de personas representa un poco más del 10 por ciento de la población estadunidense, que en 2011 se estimaba en 311.5 millones (wTO, 2014a).

${ }^{3}$ A fin de proteger a los canadienses e incrementar la seguridad mundial, el presupuesto de febrero del 2005 asignó a las fuerzas canadienses cerca de 13000000 de dólares durante los siguientes cinco años. Se trata del mayor incremento de los gastos de defensa en dos décadas y constituye la nueva imagen que Canadá proyecta al exterior (DFAIT, 2005: 28).
} 
Los tres postulados previos se resumen en defensa, comercio y desarrollo, lo que implica que, de acuerdo con la visión estratégica del gobierno canadiense, no se dispersarían esfuerzos entre múltiples actividades o entre todas las regiones. Desde luego, Estados Unidos ha mostrado su beneplácito respecto de estos tres puntos, en la medida en que la acción trilateral unificada con México resulta más que una relación económica. En efecto, se trata de una relación estratégica en la que se conforma un frente común, particularmente en dos temas que son clave para Estados Unidos relativos a la seguridad nacional y a la cuestión energética.

En cuanto a la estrategia de incrementar y diversificar el comercio, Canadá delineó trece mercados prioritarios: Estados Unidos, México, América Latina y el Caribe, la Asociación de Naciones del Sureste Asiático, Australia, Nueva Zelanda, Brasil, China, Corea, India, Japón y Rusia. A pesar de los esfuerzos realizados por las autoridades canadienses en la dirección mencionada, lo cierto es que las relaciones comerciales con algunos de estos países son mínimas o casi inexistentes.

Como se observa en el cuadro 1, a pesar de los intentos de Canadá de diversificar sus exportaciones, Estados Unidos sigue siendo su socio preferencial. La segunda y tercera posición las ocupaban Japón y Reino Unido, respectivamente, que se mantenían como socios importantes para Canadá hasta 2011. En el caso de Reino Unido, posiblemente el comercio se relacione más con un vínculo histórico de Canadá como parte de la comunidad británica.

El caso del comercio con China es destacable, ya que las exportaciones a ese país se ubicaban en el cuarto sitio en 1995 y pasaron al tercer lugar en 2011, triplicándose su volumen en dicho periodo; mientras que México, su socio comercial, no figuraba dentro de los primeros diez en 1995 y se colocó en el quinto lugar en 2011. Lo anterior nos indica que, a pesar de los esfuerzos de las autoridades canadienses por diversificar geográficamente sus intercambios, el comercio se mantiene fuertemente concentrado con Estados Unidos.

Sin duda, uno de los cambios más notables en el comercio canadiense de los últimos diez años proviene de la composición de las importaciones de Canadá por país de origen. En primer lugar, destaca la tendencia descendente de las mercaderías provenientes de Estados Unidos, que en 1995 representaban casi las dos terceras partes (el 66.81 por ciento respecto del total importado), que se reafirmó en 2001 con el 63.6 por ciento, pero hacia 2011 dicha cifra tuvo una disminución notable al representar el 49.5 por ciento; no obstante, en la estructura de las importaciones canadienses por país de origen aún prevalece una relativa alta concentración de los productos provenientes de Estados Unidos. 


\begin{tabular}{|c|c|c|c|c|c|c|c|c|}
\hline \multicolumn{9}{|c|}{$\begin{array}{c}\text { Cuadro } 1 \\
\text { EXPORTACIONES DE CANADÁ AL MUNDO }\end{array}$} \\
\hline 1995 & País & $\%$ & 2001 & País & $\%$ & 2011 & País & $\%$ \\
\hline 1 & Estados Unidos & 79.21 & 1 & Estados Unidos & 87.05 & 1 & Estados Unidos & 73.72 \\
\hline 2 & Japón & 4.60 & 2 & Japón & 2.06 & 2 & Reino Unido & 4.20 \\
\hline 3 & Reino Unido & 1.48 & 3 & Reino Unido & 1.25 & 3 & China & 3.76 \\
\hline 4 & China & 1.32 & 4 & China* & 1.06 & 4 & Japón & 2.38 \\
\hline 5 & Alemania & 1.26 & 5 & Alemania & 0.73 & 5 & México & 1.22 \\
\hline 6 & Corea del Sur & 1.04 & 6 & México & 0.68 & 6 & Corea del Sur & 1.14 \\
\hline 7 & Francia & 0.75 & 7 & Francia & 0.54 & 7 & Holanda & 1.07 \\
\hline 8 & Bélgica & 0.72 & 8 & Corea del Sur & 0.50 & 8 & Alemania & 0.88 \\
\hline 9 & Italia & 0.70 & 9 & Bélgica & 0.48 & 9 & Francia & 0.69 \\
\hline \multirow[t]{3}{*}{10} & Hong Kong & 0.67 & 10 & Italia & 0.41 & 10 & Hong Kong & 0.66 \\
\hline & Resto de países & 8.23 & & Resto de países & 5.25 & & Resto de países & 10.28 \\
\hline & Mundial & 100 & & Mundial & 100 & & Mundial & 100 \\
\hline
\end{tabular}

En abierto contraste con el retroceso de las importaciones procedentes de Estados Unidos, se observa el rápido ascenso de las provenientes de China, que en 2001 representaban sólo el 3.71 por ciento del total importado que la colocaba en la tercera posición, sin embargo, en 2011 había escalado hasta la segunda posición con un 10.8 por ciento, por lo que desplazó a Japón. La tercera posición la ocupa México con un 5.51 por ciento del total de las importaciones canadienses (como se observa en el cuadro 2), de tal forma que después de Estados Unidos, China se ha convertido en el nuevo gran socio de Canadá.

\begin{tabular}{|c|c|c|c|c|c|c|c|c|}
\hline \multicolumn{9}{|c|}{$\begin{array}{c}\text { Cuadro } 2 \\
\text { IMPORTACIONES DE CANADÁ DEL MUNDO }\end{array}$} \\
\hline 1995 & País & $\%$ & 2001 & País & $\%$ & 2011 & País & $\%$ \\
\hline 1 & Estados Unidos & 66.81 & 1 & Estados Unidos & 63.62 & 1 & Estados Unidos & 49.53 \\
\hline 2 & Japón & 5.36 & 2 & Japón & 4.27 & 2 & China & 10.8 \\
\hline 3 & Reino Unido & 2.43 & 3 & China & 3.71 & 3 & México & 5.51 \\
\hline 4 & México & 2.37 & 4 & México & 3.53 & 4 & Japón & 2.93 \\
\hline 5 & Alemania & 2.13 & 5 & Reino Unido & 3.42 & 5 & Alemania & 2.87 \\
\hline 6 & China & 2.06 & 6 & Alemania & 2.33 & 6 & Reino Unido & 2.32 \\
\hline 7 & $\begin{array}{l}\text { Reimportaciones } \\
\text { (Canadá) }\end{array}$ & 1.72 & 7 & $\begin{array}{l}\text { Reimportaciones } \\
\text { (Canadá) }\end{array}$ & 2.07 & 7 & Corea del Sur & 1.48 \\
\hline 8 & Italia & 1.45 & 8 & Francia & 1.61 & 8 & Francia & 1.24 \\
\hline 9 & Corea del Sur & 1.42 & 9 & Corea del Sur & 1.34 & 9 & Argelia & 1.23 \\
\hline 10 & Francia & 1.39 & 10 & Taiwán & 1.29 & 10 & Italia & 1.14 \\
\hline & Resto de países & 12.87 & & Resto de países & 12.82 & & Resto de países & 20.95 \\
\hline & Mundial & 100 & & Mundial & 100 & & Mundial & 100 \\
\hline
\end{tabular}


Como hemos visto, los esfuerzos de Canadá por diversificar sus relaciones comerciales no han tenido los resultados esperados, pero lo que sorprende es la nueva relación comercial con un socio emergente como China. ¿Qué sucede en estos intentos de diversificación? Canadá ha firmado muchos acuerdos de libre comercio con varios países, sin embargo, su comercio con otras naciones emergentes como Corea del Sur, Brasil o India no logra despegar.

En el TLCAN, el nivel de los flujos comerciales había mostrado un crecimiento importante, hasta cierto punto, sin embargo, las corrientes de comercio comenzaron a mostrar cambios a partir de 2001, que no se atribuyen a modificaciones internas de los tres países socios, sino que se relacionan específicamente con las transformaciones impulsadas por China, en su condición de nuevo actor de la escena internacional. Ciertamente, el complejo proceso de cambios productivos, comerciales e institucionales experimentados por la economía china en los últimos veinte años ha favorecido la productividad y competitividad de sus manufacturas y exportaciones. Parece evidente que la elevada productividad y competitividad de las exportaciones chinas de varias formas han influido en la evolución y redefinición de las relaciones comerciales entre los tres países socios en el TLCAN.

\section{El impacto de la competitividad de China en el comercio de Canadá y Estados Unidos}

El andamiaje comercial que se consolidó en 1994 con la firma del TLCAN al cabo de seis años comenzó a tomar un rumbo diferente, no tanto por un cambio en la relación histórica, comercial y preferencial entre Estados Unidos y Canadá, sino atribuible a lo que denominamos "efecto chino", definido como la capacidad productiva y competitiva de esta nación asiática que le ha permitido imponerse en poco tiempo como segundo socio comercial de Canadá, Estados Unidos y también de México.

En 1997, China no tenía un peso importante en el comercio de Canadá y de Estados Unidos. Como se muestra en la gráfica 3, es justamente a partir de 2001 cuando las exportaciones chinas comenzaron a mostrar una tendencia ascendente hacia Estados Unidos. Hasta el año 2000, las importaciones provenientes de México y Canadá eran más importantes que las de China, sin embargo, en un lapso de siete años, estas últimas cobraron una importancia nunca vista y con un margen amplio en comparación con sus socios comerciales.

La rápida penetración de las manufacturas chinas ha sido tal que en el contexto de la crisis financiera y productiva de Estados Unidos en 2008 y 2009, y después de un menor crecimiento, las importaciones provenientes de China de nueva cuenta se 
recuperaron en el 2010 y alcanzaron los 383000000001 de dólares. En tan sólo diez años, las importaciones estadunidenses provenientes de esta nación asiática se triplicaron si consideramos que en 2000 sumaron un poco más de cien mil millones de dólares.

\section{Gráfica 3}

IMPORTACIONES DE ESTADOS UNIDOS PROCEDENTES DE MÉXICO, CANADÁ Y CHINA

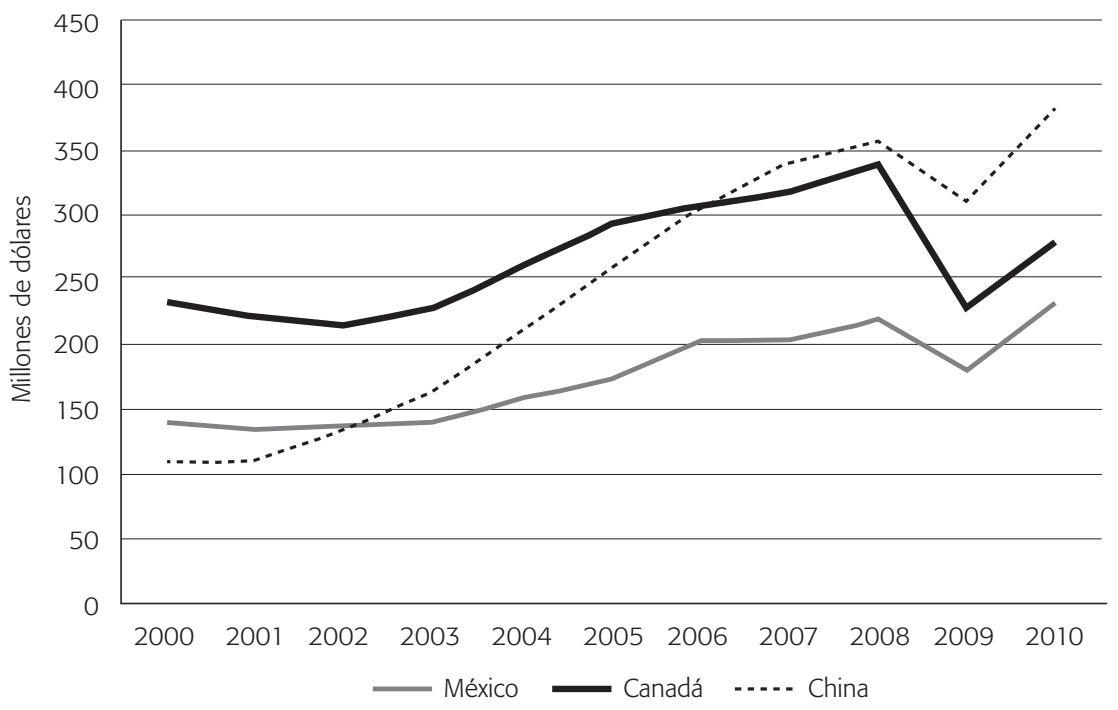

Fuente: OMC, 2011.

En cuanto a las exportaciones de Estados Unidos hacia China, advertimos que no muestran el mismo dinamismo ni el nivel que las importaciones, como se observa en la gráfica 4. Si bien es cierto que las exportaciones estadunidenses a China se han incrementado más de 500 por ciento, al pasar de 2000 a 2010 de 16300000000 de dólares a 91900000000 de dólares, también lo es que las exportaciones hacia los mercados de sus dos socios comerciales de Norteamérica han mostrado un comportamiento ascendente, con excepción de 2009, cuando descendieron, pero al año siguiente se recuperaron. En estas condiciones, cabe destacar que las exportaciones de Estados Unidos a China no registraron un impacto negativo importante durante la crisis financiera de 2008. Este comportamiento contrasta con la declinación fuerte que mostraron las exportaciones de Canadá y México, sus dos socios preferenciales de la región. 


\section{Gráfica 4}

EXPORTACIONES DE ESTADOS UNIDOS HACIA MÉXICO, CANADÁ Y CHINA

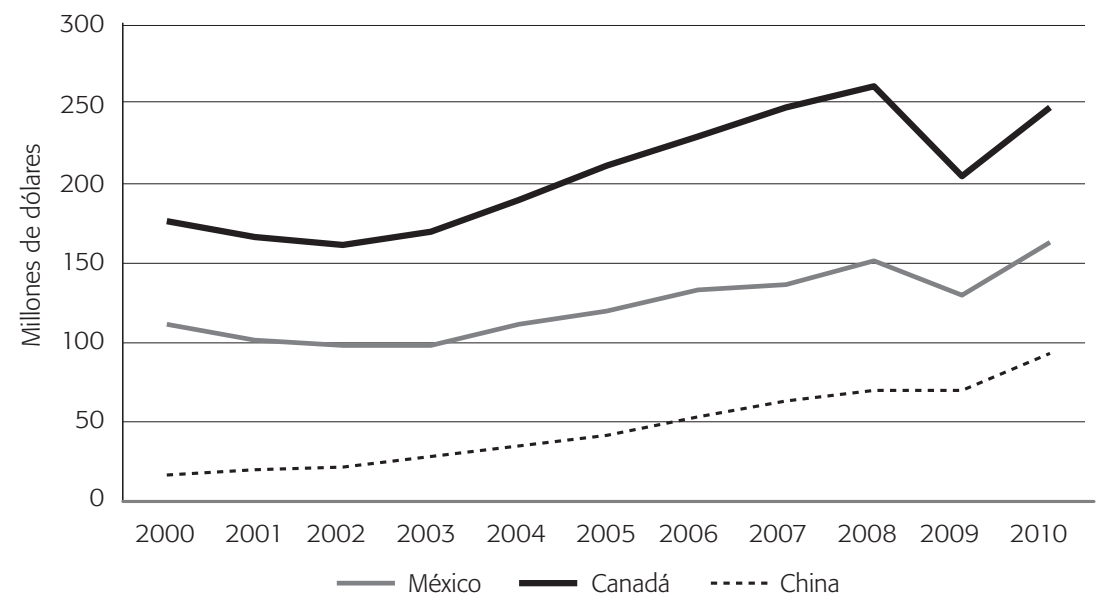

Fuente: OMC (2011).

No obstante que geográficamente China se encuentra a más de 12000 kilómetros de Estados Unidos y que aparentemente no presenta una cercanía cultural en la manera de hacer negocios, advertimos que los arreglos institucionales formales e informales que han pactado Estados Unidos y China son muy fuertes, y con reglas del juego claras.

En cuanto al comercio de Canadá con China, observamos que también ha registrado cambios importantes. Como se observa en la gráfica 5, las importaciones de Canadá provenientes de México eran ligeramente superiores a las de China en 1995; sin embargo, al igual que en el caso de Estados Unidos, a partir de 2001 se presenta un despliegue importante del comercio con China si lo comparamos con el comportamiento de las importaciones mexicanas, de tal forma que hacia 2011 el valor de las mercaderías provenientes de China fueron dos veces más elevadas que las procedentes de México.

Un fenómeno especialmente importante se relaciona con la crisis financiera desatada en el mercado de los créditos hipotecarios en Estados Unidos, que tuvo un efímero efecto en China. Esto se debió a que el principal mercado externo para China sigue siendo la región asiática. En efecto, en 2011, las exportaciones chinas a Hong Kong, Japón y Corea del Sur suman más del 26.3 por ciento, una cifra mayor al 17.1 por ciento que representa el mercado de Estados Unidos y el 18.1 por ciento que significan los 27 países de la Unión Europea. Como se observa, el mercado norteameri- 
cano ciertamente relevante no es mayor que la cuota que representan para China el conjunto de los mercados de sus vecinos asiáticos.

Por su parte, las importaciones chinas procedentes de Japón, Corea del Sur y Taiwán suman 27.7 por ciento del total de las importaciones que contrastan con el 7. 1 por ciento que representan las importaciones procedentes de Estados Unidos y el 12.2 por ciento de la Unión Europea (WTO, 2013). En estas condiciones, podemos considerar que la desaceleración que ha presentado la economía china en los últimos años se debe más a presiones internas de sobrecalentamiento de la economía que a factores externos.

\section{Gráfica 5}

IMPORTACIONES DE CANADÁ PROCEDENTES DE MÉXICO Y CHINA

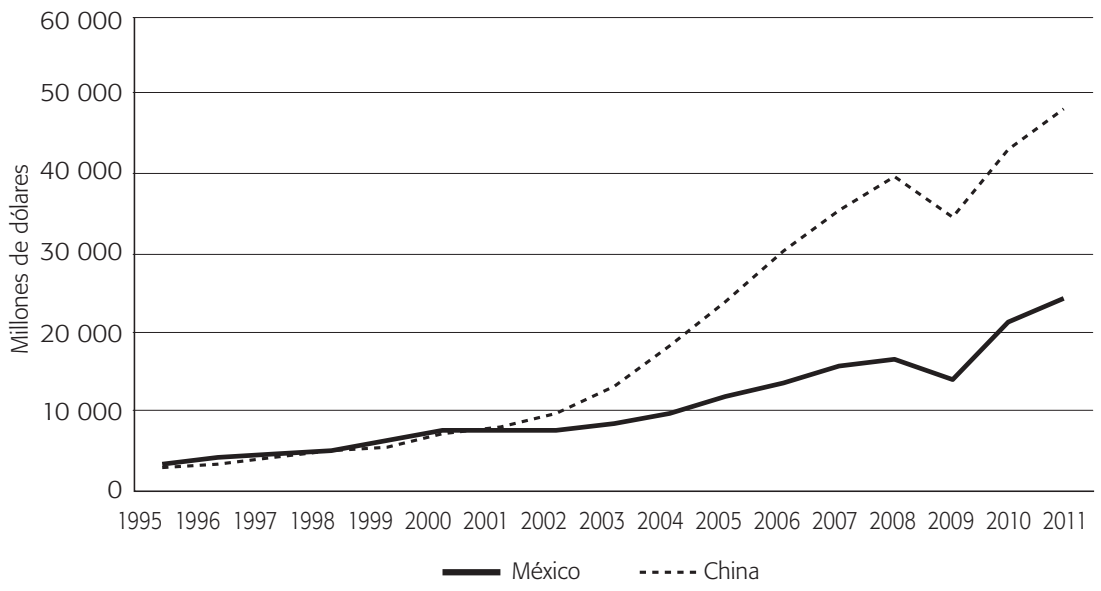

Fuente: Industry Canada (2012).

Históricamente, Canadá ha construido y sostenido un fuerte vínculo comercial con Estados Unidos, el cual representa una sólida ventaja comercial en la exportación de bienes y servicios respecto de la competencia china. Conviene recordar que para esta nación asiática la relevancia del mercado norteamericano radica en su condición de principal destino de sus exportaciones manufactureras, en tanto que, como ya vimos, una gran parte de las importaciones de China no provienen de Estados Unidos, sino de otros mercados, como los de Asia Pacífico y de América Latina.

En la actualidad, Canadá ya no es el principal socio comercial de Estados Unidos, pues fue desplazado por China a partir del 2007, como se muestra en la gráfica 3; no obstante, una pregunta que surge es qué impacto tiene para Canadá este cambio 
en su posición comercial con Estados Unidos. ¿Qué factores generaron este parteaguas para que a partir del 2006 se perdiera el dinamismo de las exportaciones canadienses? ¿Tiene efectos negativos en su comercio con Estados Unidos?

Desde el punto de vista institucionalista, el cual subraya la importancia del papel que cumplen las instituciones políticas y económicas en el desarrollo de largo plazo y el desempeño macroeconómico de los países, las reformas institucionales en el sector agrícola, los incentivos conducidos por el Estado en materia de inversión extranjera directa y el desarrollo e innovación tecnológica, etc., por sí mismas establecieron un parteaguas en la trayectoria económica de China y se reflejaron en su peso comercial en la economía mundial durante los últimos años.

En esta perspectiva, el efecto del comercio chino referido al impacto de la alta competitividad de las manufacturas elaboradas en China surge a raíz de una serie de reformas económicas a las que se sujetó, a finales de los setenta. En este contexto, nos parece que el curso ascendente de la economía china en términos de crecimiento del producto, la inversión doméstica y extranjera, y sus logros en competitividad, convirtieron a China en el nuevo jugador internacional que se confirmó plenamente con su ingreso a la omc en 2001.

El ingreso de China a la principal organización multilateral creada después de la guerra, la OMC, intensificó la instrumentación de una serie de cambios institucionales y ratificó, desde el punto de vista de los organismos multilaterales del comercio, la credibilidad en la aplicación de las reformas emprendidas desde los años setenta (Estrada, 2006: 379). También hay que considerar que un requisito clave del éxito de las reformas económicas y sociales ha sido el papel activo y orientador de largo plazo del Estado en las transformaciones, así como su compromiso para aplicar a tiempo las correcciones y los cambios precisos de acuerdo con lo que demandaban las reformas económicas (Tarassiouk, 2006: 407).

El bajo nivel de los costos laborales, derivado de la relativa abundancia en mano de obra calificada y no calificada que ha caracterizado a las primeras etapas del modelo de desarrollo de capitalismo dirigido por el Estado chino, representa uno de los principales determinantes de su competitividad, mas no es el único. Sin embargo, esta ventaja comparativa tiende a disminuir frente a un constante ascenso de los salarios reales en las principales zonas y ramas industriales de su aparato productivo. Aunado a lo anterior, es probable que el nuevo marco normativo haya repercutido en los bajos costos de transacción derivados de los contratos, entre otros, de tal forma que si a los bajos costos de producción se agrega el efecto de los bajos costos de transacción -que además de dar certidumbre aseguran las inversiones- no queda duda de que ambos configuran una poderosa estructura de incentivos a favor de la producción y las inversión interna y extranjera en esta nación de Asia Pacífico. 
En diferentes momentos, las autoridades de China promovieron y establecieron un nuevo marco normativo económico e institucional internamente y en sus relaciones con la economía mundial, que en el largo plazo determinaron las reglas del juego económico, las que a su vez propiciaron y facilitaron el papel de nueva potencia económica en los mercados internacionales sellados por las tendencias de la globalización (González, 2012: 125-126). El compromiso institucional de la economía china marca la diferencia en términos de su alto dinamismo y la adquisición de ventajas competitivas y comparativas que explican su meteórico ascenso como jugador mundial. En estas condiciones, su poderío comercial y manufacturero ha generado grandes impactos en los flujos internacionales y en los bloques de comercio regional, confirmado con el éxito comercial chino frente a México y Canadá en la carrera por el mercado estadunidense, aunque se diría que el éxito frente a Canadá aún no es rotundo.

El crecimiento del PIB de China como resultado del éxito de las reformas emprendidas, así como de su ingreso a la OMC, la ha convertido en el país con las reservas internacionales más grandes a nivel mundial y que haya adquirido gran parte de los bonos de deuda del Tesoro estadunidense, de tal forma que es el principal acreedor de la deuda de Estados Unidos. ${ }^{4}$ Sin duda alguna, la posición acreedora de China le permite un margen de negociación significativo en los temas comercial y cambiario que conduce a arreglos no formales en materia comercial. El financiamiento chino y su calidad de primer socio comercial explican, en principio, por qué Estados Unidos conserva una relación minuciosa y prudente con esa nación.

Esta situación permite comprender la persistencia de una política suave de baja presión por parte del gobierno estadunidense en el tema de la revaluación del yuan frente al dólar. En estas condiciones, la supuesta "guerra de divisas" que mantienen ambos países desde 2010 es sólo en una expresión y nada más. Ciertamente, a ninguno de los dos socios le conviene romper el particular entramado de reglas del juego comerciales y financieras que ambos protagonizan. No se olvide el fuerte vínculo financiero, toda vez que China es el principal acreedor de los bonos del Tesoro estadunidense. En todo caso, China regresaría de golpe todo el papel en deuda de los bonos de Estados Unidos y le exigiría hacer cuentas, lo que conduciría a una caída abrupta del dólar y de otras divisas; un escenario que tampoco le conviene a China,

\footnotetext{
${ }^{4}$ A fines de 2012, las reservas internacionales de China alcanzaron los 3.3 billones de dólares, el doble de la reserva global del oro, según los datos de Bloomberg. Desde 2004, el precio del oro subió 263 por ciento, hasta 1580 dólares por onza troy; las reservas internacionales de China crecieron un 721 por ciento en el mismo periodo. El oro representa apenas un 2 por ciento en las reservas internacionales de China, en comparación con un 70 por ciento en Estados Unidos y Alemania, pero, por el volumen de reservas, China ocupa el primer lugar del mundo, seguida de Japón, Arabia Saudita, Rusia y Suiza. China controlaba, a fines de 2012, 30.2 por ciento de las reservas internacionales a escala global, en relación con un 14 por ciento a principios de 2004. Dos tercios de estos activos chinos están nominados en dólares y otra cuarta parte, en euros (RIA Novosti, 2013).
} 
dado el estatus de cliente preferencial que representa el mercado de Estados Unidos para sus exportaciones.

En su relación con Estados Unidos, Canadá se apegó a una serie de acuerdos institucionales y no institucionales enmarcados en reglas escritas y no escritas, fincados en la política de buena vecindad que implica la cercanía geográfica, así como en las similitudes culturales, étnicas, políticas, etc., entre ambas naciones. Estos factores institucionales en el largo plazo desembocaron en una relación comercial y económica intensa y estructural. En efecto, la relación bilateral entre Estados Unidos y Canadá está tan arraigada, que la competencia china no la ha quebrantado del todo, pero sí la ha desplazado de su posición exportadora número uno con su vecino país del Sur.

Reglas diferentes, cambio institucional y protección de los derechos de propiedad son elementos importantes en la construcción de la relación comercial entre estos dos países en el siglo xx y lo que va del xxI, que encuentra puntos de apoyo decisivos en la cercanía geográfica, la cultura y una política internacional de seguridad compartida.

A pesar de que a partir de 2006 el valor de las exportaciones de bienes de China a Estados Unidos es mayor que el de las exportaciones de Canadá y nada parece debilitar esta posición, vale la pena revisar la estructura del comercio canadiense-estadunidense, pues no son mercados competitivos.

No se diría lo mismo respecto de México, que sí tiene que hacer frente a la competencia china en la carrera comercial en el mercado estadunidense, especialmente porque en su estructura de exportaciones encontramos que el automotriz y la electrónica son sectores claramente competitivos respecto de las exportaciones de China en este mercado. La competencia con las manufacturas chinas en el mercado estadunidense no sólo enfrenta los bajos costos de mano de obra, calidad y precio, sino que además el contenido tecnológico y la estructura comercial recae en las manufactureras similares. Asimismo, gran parte de las manufacturas mexicanas exportadas a Estados Unidos llevan ensamblados insumos de origen chino.

\section{¿HaCia dónde VA LA Relación CanadÁ-Estados Unidos?}

La relación de Canadá con Estados Unidos es particular, y a pesar de los cambios detectados en los últimos años en materia comercial de este último con China, pareciera que éstos no afectan de forma determinante la dinámica comercial de Canadá por tres razones: la primera radica en que Canadá ha sido un proveedor histórico de Estados Unidos de recursos naturales dado que es uno de los principales productores de minerales en el mundo. Este país es el principal productor de níquel, potasio y 
asbesto; también figura entre los primeros en el caso del cobre, el zinc, el plomo y el uranio. En consecuencia, las exportaciones canadienses de estos productos mineros al mercado estadunidense no enfrentan la competencia de China que, a su vez, es uno de los países de alta demanda de estos productos en el mercado mundial.

También hay que considerar que gracias a la abundancia de energía hidroeléctrica, los procesos industriales de reducción electrolítica de varios metales se realizan en Canadá, como el níquel, cobre, zinc, plomo y aluminio, lo cual agrega ventaja a estos productos de exportación. Además, la producción de algunos de estos minerales es controlada por empresas estadunidense y esto asegura el aprovisionamiento al mercado de dicho país.

Conviene revisar algunos indicadores sobre las tendencias del comercio canadiense con Estados Unidos. En 2009, las exportaciones canadienses de productos agrícolas y pesqueros representaron el 9 por ciento del total, los productos forestales el 5.1 por ciento, los productos energéticos 24.5 por ciento y la suma de estos tres tipos de bienes, el 39.5 por ciento del total de las exportaciones, que, como hemos afirmado, no están sujetas por su naturaleza a la competencia de las exportaciones de China en los mercados de Estados Unidos.

En 2102, del total de las exportaciones, los productos agrícolas constituyeron el 5.7 por ciento, los productos energéticos el 24.5 por ciento, los minerales metálicos y no metálicos el 15 por ciento, los productos forestales el 6.3 por ciento, de tal forma que estos cuatro sectores representan más del 50 por ciento de las exportaciones totales de Canadá. Conviene señalar que las exportaciones de vehículos y autopartes representaron para ese mismo año el 14 por ciento del total exportado, un sector que estaría bajo la presión de la competencia china (Statistics Canada, 2013).

Actualmente, Canadá se mantiene como un proveedor clave de Estados Unidos en su estructura de importaciones basada en los recursos naturales. Como se muestra en la gráfica 6, sólo después del conjunto de países que forman la Unión Europea, Canadá es el principal exportador de hierro y acero a Estados Unidos, con una cuota de participación en 2011 del 15.3 por ciento contra un 6.7 por ciento de China; incluso México exporta a Estados Unidos más hierro y acero que China, según se indica en la gráfica 6, relativa a las importaciones de minerales por Estados Unidos.

Como se observa en el cuadro 3, en el 2000 China tenía poca participación en la importación de recursos naturales de Estados Unidos. El valor de los combustibles importados en ese año era del 3.5 por ciento del total correspondiente a este renglón; sin embargo, en 2010, prácticamente se había duplicado al sumar 6.9 por ciento de la importación total de combustibles por Estados Unidos. Se consideraría una cifra poco significativa si se comparan con las exportaciones chinas al mercado estadunidense de bienes intensivos en tecnología, como la maquinaria en general y las pren- 
das de vestir (el 13.2 por ciento). Si comparamos en ese mismo año las exportaciones canadienses a Estados Unidos, observamos que sus mayores exportaciones se componen de combustibles (el 24.5 por ciento) y hierro y acero (el 15.9 por ciento).

\section{Gráfica 6}

IMPORTACIONES DE ESTADOS UNIDOS EN HIERRO Y ACERO, 2011

(participación por región/país)
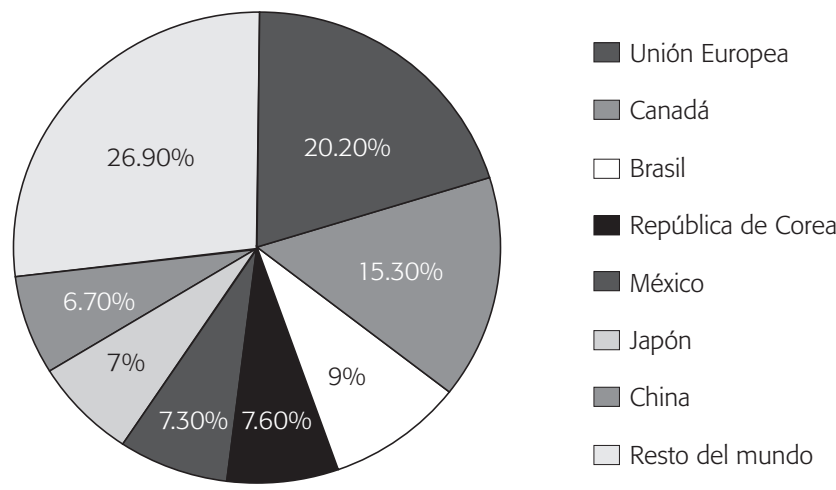

Fuente: WTO, 2012.

Hacia 2011, las exportaciones chinas a Estados Unidos se concentran en maquinaria, al representar el 47 por ciento del total importado de esta categoría de bienes; textiles, 37.7 por ciento, y prendas de vestir cuya cuota aportó el 39.4 por ciento del total importado por Estados Unidos en este tipo de bienes.

Por su parte, las exportaciones canadienses se concentran principalmente en combustibles que significaron el 22.6 por ciento del total importado en este renglón por Estados Unidos; los productos agrícolas aportan el 20.8 por ciento, mientras que el hierro y el acero significaron el 15.3 por ciento de la cuota total de estos productos o mercancías básicas (commodities) importadas por el mercado estadunidense en 2011.

Hay un cambio interesante en la estructura de importaciones de Estados Unidos respecto de los químicos provenientes de China que en 2010 representaban el 45 por ciento del total importado, contrastante con la cuota canadiense de 1.5 por ciento de la cuota total de este insumo. También cabe señalar una disminución sensible de las importaciones de hierro y acero provenientes de Canadá que disminuyó notablemente del 19 por ciento en 2010 al 15.6 por ciento en 2011, equiparable al nivel que tenía en 2000. 
Por otra parte, se diría que China avanzó si consideramos que en 2000 su cuota representaba apenas el 3.5 por ciento y aumentó al 6.3 en 2010 y alcanzó el 6.9 por ciento de la cuota total importada en 2011.

\begin{tabular}{|c|c|c|c|c|c|c|}
\hline \multicolumn{7}{|c|}{$\begin{array}{c}\text { Cuadro } 3 \\
\text { IMPORTACIONES DE ESTADOS UNIDOS PROVENIENTES DE CANADÁ Y CHINA } \\
\text { (porcentaje del total por tipo de mercancías) }\end{array}$} \\
\hline \multirow[b]{2}{*}{ Productos } & \multicolumn{3}{|c|}{ Canadá } & \multicolumn{3}{|c|}{ China } \\
\hline & 2000 & 2010 & 2011 & 2000 & 2010 & 2011 \\
\hline Alimentos & n.d. & 19.2 & 18.9 & n.d & 5.5 & 5.4 \\
\hline Agrícolas & n.d. & 22 & 20.8 & n.d & 5.6 & 5.5 \\
\hline Químicos & n.d. & 1.7 & n.d & n.d & 45.4 & n.d \\
\hline Combustibles & 24.5 & 23.2 & 22.6 & 0.5 & n.d & n.d \\
\hline Hierro y acero & 15.9 & 19 & 15.3 & 3.5 & 6.3 & 6.9 \\
\hline $\begin{array}{l}\text { Máquinas de oficina } \\
\text { y equipo de telecomunicaciones }\end{array}$ & 7.4 & 1.7 & 1.6 & 10.2 & 45.4 & 47.7 \\
\hline Textiles & 12.5 & 6.3 & 6.1 & 12 & 37.4 & 37.7 \\
\hline Prendas de vestir & 3 & 0.8 & 0.8 & 13.2 & 40.9 & 39.4 \\
\hline Autopartes & n.d. & 25.3 & 24.3 & n.d & 3.1 & 3.4 \\
\hline
\end{tabular}

Como se observa en el cuadro 3, las exportaciones chinas a Estados Unidos en los sectores intensivos en mano de obra e intensivos en capital no representan una seria amenaza para la economía canadiense, orientada -como ya hemos visto- en buena medida a la producción y exportación de recursos naturales, lo que indicaría que en bienes primarios Canadá y China no son países competitivos en el mercado estadunidense.

En realidad, las ventajas del comercio de Canadá frente a China en aquel mercado radican en la estructura de sus exportaciones, centrada específicamente en la rama energética y los bienes basados en la explotación de sus recursos naturales, cuyo suministro reporta ventajas absolutas en los costos de transporte por la cercanía geográfica y la infraestructura de comunicaciones.

En resumen, afirmamos que el comercio canadiense, dada la estructura de las exportaciones a Estados Unidos, caracterizada por una elevada concentración en energéticos, minerales y otros bienes básicos, mantiene fuertes ventajas derivadas de 
la abundancia de recursos naturales y bajos costos de transportación por la cercanía geográfica con el mercado del vecino país del Sur.

En este sentido, hay indicios de que la economía canadiense seguirá ocupando un sitio de socio preferencial y estratégico con Estados Unidos, y en el corto y mediano plazos no se advierten riesgos importantes de la competencia de China, cuyas principales exportaciones se colocan en los mercados de bienes manufacturados y de capital de alta tecnología.

La situación de México es distinta y habría que tomar muy en serio la competencia comercial china. A diferencia de Canadá, el sector exportador mexicano mantiene un fuerte vínculo comercial en la exportación de manufacturas con Estados Unidos que, por cierto, tienen una importante incorporación de insumos provenientes de China. De hecho, la competencia de los productos chinos ha desplazado a los mexicanos desde el 2002, y no sólo en industrias intensivas en mano de obra, sino también en aquellas con mayor contenido tecnológico (Guzmán y Toledo, 2005: 95).

Los recursos naturales provenientes de Canadá son tan importantes para Estados Unidos, que un intento por llegar a un consenso sobre su aseguramiento fue el proyecto de Acuerdo para la Seguridad y Prosperidad de América del Norte (ASPAN), mediante el cual los gobiernos de México, Estados Unidos y Canadá se orientaban a establecer un pacto que reconociera un equilibrio entre la seguridad en el aprovisionamiento y la seguridad en el acceso a los recursos naturales.

Este proyecto trilateral incluía normas relacionadas con los precios y las dificultades bilaterales, una tarifa externa común y el compromiso de volver más eficiente la red de transporte para convertir a la región en una zona más competitiva en términos de inversiones y producción; sin embargo, este acuerdo, cuyo principio era "la seguridad y la prosperidad dependiente y complementaria" no se consolidó; al final de cuentas, Estados Unidos tiene asegurados los recursos naturales de Canadá a través de sus inversiones y el control de importantes activos en diversas ramas de la producción de bienes y servicios en la economía canadiense.

La segunda razón es que Canadá y México son los principales proveedores de energéticos a Estados Unidos, por lo que la seguridad energética resulta entonces una prioridad para el socio mayor. Ciertamente, la producción de gas y petróleo ha declinado en Estados Unidos, ${ }^{5}$ Canadá y México; no obstante, los dos últimos países tienen un gran potencial para desarrollar el crecimiento de estos sectores, producir su propio consumo y exportar. La importante disponibilidad de recursos energéticos con los que cuenta Canadá -considerado el segundo país en el mundo en términos de

\footnotetext{
${ }^{5}$ En 1991, Estados Unidos producía 9.1 millones de barriles de petróleo diarios y en 2005 la producción se redujo a 7.61 millones de barriles diarios, llevándolo a ocupar el cuarto lugar a nivel mundial (Cedefna, 2006).
} 
reservas probadas-, ha despertado el interés por realizar sustanciales inversiones por parte de países como China, que no dispone del recurso, y que por las características de su actual desarrollo necesita cantidades considerables de hidrocarburos.

Contrariamente a lo que se afirma, el gobierno del primer ministro Stephen Harper ha incentivado la explotación de las arenas bituminosas, a partir de las cuales se extrae el petróleo en la provincia de Alberta, lo que tiene efectos perversos para la economía de ese país, ya que encierra a Canadá en un modelo de desarrollo dependiente del carbón que perjudica la innovación de escalar industrias de desarrollo durable. ${ }^{6}$

Esta industria se beneficia de un importante apoyo político e incentivos por parte del gobierno de Harper, particularmente desde que el gobierno conservador tomó el poder, al favorecer un incremento en la producción y exportación, así como en la creación de varios oleoductos que ha conducido al país a experimentar un auge petrolero no planeado e incontrolable. Las empresas petroleras en suelo canadiense, principalmente las transnacionales extranjeras, consideran duplicar su producción a finales de este decenio, hasta llegar a los 3.5 millones de barriles por día (Shields, 2013).

Un grupo importante es la Asociación Canadiense de Productores de Petróleo, que aglutina a los productores de gas y petróleo que producen el responsables del 95 por ciento de ambos insumos, genera un ingreso anual de cien mil millones de dólares y emplea a más de medio millón de canadienses. En esta asociación se encuentran empresas estadunidenses importantes a nivel mundial, como Shell, Chevron (dueña de lo que anteriormente fue Standard Oil of California y Gulf Oil), Exxon (dueña del otrora Esso), así como de gran parte de la Imperial Oil, la empresa más importante de petróleo y gas de Canadá, entre otras (CAPP, 2007).

La tercera razón es que entre los compromisos entre Canadá y Estados Unidos se encuentran los acuerdos suscritos en materia de defensa y seguridad nacional que permiten hacer frente común a las amenazas externas. Esta tendencia la ilustran diversos pactos, como la "Declaración de la frontera inteligente" de 2001, ${ }^{7}$ la constitución del Grupo de Planificación Binacional en 2002, la "Declaración conjunta sobre seguridad y prosperidad comunes" en 2004 y en 2005 el "Reporte conjunto", presentado por el Consejo de Relaciones Exteriores de Estados Unidos, el Consejo Canadiense de Ejecutivos y el Consejo Mexicano de Asuntos internacionales.

Para Estados Unidos, su relación con Canadá se mantendrá estable y segura en materia comercial y política. Es cierto que China ocupa ya el primer lugar en el comercio

\footnotetext{
${ }^{6}$ La producción de petróleo a partir de las arenas bituminosas es altamente contaminante, ya que además de implicar una tala enorme de árboles, la extracción del bitumen genera contaminación de ríos y un enorme consumo de agua.

${ }^{7}$ En virtud de que el intercambio comercial es muy intenso en estos dos países, y con el fin de promover la seguridad en la frontera y agilidad de los intercambios comerciales, la frontera inteligente consiste, entre otras cosas, en que oficiales de migración estadunidenses estén directamente en las plantas productoras canadienses para certificar in situ que las mercancías que se embarcan son las correctas (Montero, 2002).
} 
de ambos países, pero para Canadá este cambio no es un problema en términos comerciales si se considera (como hemos visto) que las mercancías exportadas no entran en competencia por tratarse de mercados diferenciados; sin embargo, el reto para Canadá es que los acuerdos institucionales vigentes con Estados Unidos generen incentivos que refuercen o reafirmen la actual estructura del comercio y la industria canadienses, y frenen así la puesta en marcha de políticas dirigidas a la diversificación geográfica y por tipo de bienes de la estructura de sus exportaciones. Una falta de iniciativa al respecto o la posible parálisis en esta materia por parte de las autoridades canadiense sí representaría un problema mayor.

\section{FUENTES}

Ayala, José

2011 Instituciones y economía. Una introducción al neoinstitucionalismo, México, FCE.

CAmeron, Duncan

2000 "Privatización y ajuste estructural", en T. Gutiérrez Haces, coord., Canadá, un Estado posmoderno, México, Plaza y Valdés.

capp (Canadian Association of Petroleum Producers)

2007 “CAPP Stewardship Repport”, en <http:/ / stewardship.capp.ca/pdfs/CAPP_ 2007_Stewardship_Report.pdf>.

\section{CEDEFNA}

2006 "Boletín informativo" (mayo), en <http: / / csh.izt.uam.mx/ cen_doc/ cedefna>.

\section{Christie, KeITH}

2002 “Discurso pronunciado en el seminario 'Fronteras reales/fronteras simbólicas'”, México, 25-27 de febrero.

Correa, María Antonia y Diana Villarreal

2007 "Grupos empresariales de Monterrey y su red en América del Norte", en Delia Montero y Antonia Correa, coords., América del Norte: una integración excluyente, México, uAm/ Plaza y Valdés.

\section{DeBLOCK, CHRISTIAN}

2012 "Les relations commerciales entre le Canada et les États-Unis à l'heure des 
trois 'd'”, Centre d'Études sur l'Intégration et la Mondialisation, Montreal, Universidad de Quebec, en <www.ceim.uqam.ca $>$.

DFait (Department of Foreign Affairs, Trade and Development Canada)

2005 “Declaración de política internacional de Canadá. Nuestro rol internacional: un sentimiento de orgullo e influencia en el mundo", en <http: / / www.international.gc.ca / international/index.aspx?lang=eng $>$.

ESTRADA, JosÉ LUIS

2006 "El crecimiento económico de la República Popular China, a la luz de la experiencia de desarrollo asiático", en China en el siglo xxı. Economía, política y sociedad de una potencia emergente, México, unAm/Miguel Ángel Porrúa, pp. 363-388.

GONZÁlEZ, JUAN

2012 "El proceso de reforma económica de China: nuevas enseñanzas para México", en José Luis Calva, coord., Estrategias económicas exitosas en Asia y América Latina. Análisis estratégico para el desarrollo, vol. 2, México, Juan Pablos/Consejo Nacional de Universitarios, pp. 123-151.

Guzmán, Alenka y Alejandro Toledo

2005 "Competitividad manufacturera de México y China en el mercado estadunidense", Economía UnAM vol. 2, núm. 4 (enero-abril), pp. 94-137.

INDUSTRY CANADA

2012 "Trade Data Online. Canadian Trade by Industry (NAICs Codes)", en <http:/ / www.ic.gc.ca/eic/site/tdo-dcd.nsf/eng/Home>.

1998 http:/ / strategis.ic.gc.ca/epic/ site/ auto-auto.nsf/fr/am01165f.html.

\section{LALIBERTÉ, LuCILE}

1992 "La mondialisation et le bilan des investissements internationaux du Canada, 1950 a 1992", Statistiques Canada, doc. 6, núm. 67-202-ХРв.

Marcial, Aurora

2013 "Reestructuración productiva, crisis y tendencias en la industria automotriz en México y Canadá, 2001-2010”, en Delia Montero y Raúl Rodríguez, coords., Canadá: seguridad nacional, cambio económico e identidades. Una mirada desde América Latina, Bogotá, Universidad del Rosario-UAM, pp. 179-194. 


\section{MCCORMICK, KEN}

2006 Veblen in Plain English. A Complete Introduction to Thorstein Veblen's Economics, Nueva York, Cambria Press.

Montero, Delia

2002 "Interprovincial Trade and Intelligent Borders in Canada", Voices of Mexico no. 61, octubre-diciembre, México, pp. 64-68.

NiOSI, JORGE

1982 Les multinacionales canadiennes, Montreal, Boréal Express.

omc (Organización Mundial de Comercio)

2011 “Comercio de mercancías por regiones 2000-2010. Estadísticas de comercio internacional", en <http: / / www.wto.org/spanish/res_s/statis_s/its2011_s/its>.

PINO, ENRIQUE

2013 "El impacto de China en el TLCAN. Los límites de la integración de Canadá, México y Estados Unidos", en Delia Montero y Raúl Rodríguez, coords., Seguridad nacional, cambio económico e identidades. Una mirada desde América Latina, Bogotá, Universidad del Rosario/ UAM Iztapalapa.

RIA Novosti

2013 "Las reservas internacionales de China duplican la reserva mundial del oro", RIA Novosti, Moscú, 4 de marzo, en <http:/ / sp.ria.ru/economy/20130304/ 156541871.html>.

\section{SeCretaría de ECONOMía}

2014 "Información estadística y arancelaria", en <http://www.economia.gob. $\mathrm{mx} /$ comunidad-negocios / comercio-exterior / informacion-estadistica-yarancelaria $>$.

Secretaría de Relaciones Exteriores

2010 Boletín, en <http:/ / portal.sre.gob.mx/ canada>.

SHIELDS, AleXANDre

2013 "Les sables bitumineux, un piège coûteux. L'industrie aurait des effets pervers sur l'économie canadienne, révèle une étude", Le Devoir, 21 de febrero. 


\section{Statistics CANADA}

2013 "Le commerce international de marchandises du Canada", cuadro 228-0059, 2 de mayo, en <http: / / www.statcan.gc.ca/pub/65-001-x/2013003/t004-fra.htm>.

\section{TARAssiouk, AleXANDER}

2006 "Hacia un análisis comparativo de las reformas económicas en Rusia, China y Polonia (lecciones de pragmatismo económico para los países emergentes y en transición)", en China en el siglo XXI. Economía, política y sociedad de una potencia emergente, México, Miguel Ángel Porrúa/UAM, pp. 389-410.

\section{World Trade Organization (wTo)}

2014a "Trade Profiles: Canada", en <http://stat.wto.org/CountryProfile/wsDBCountryPFView.asp ?Language $=$ E\&Country $=$ CA, US $>$.

2014b “Trade Profiles", en <http:/ / stat.wto.org/CountryProfile/wsDBCountryPFView. asp ? Language $=\mathrm{E} \&$ Country $=\mathrm{CA}, \mathrm{MX}, \mathrm{CN}, \mathrm{US}>$.

2013 "China. Basic Indicators and Others", en <http: / / stat.wto.org/CountryProfiles/CN_e.htm>.

2012a "International Trade Statistics 2012", en <http:/ / www.wto.org/english/res_e/ statis_e/its2012_e/its12_merch_trade_product_e.htm>.

2012b "International Trade Statitics 2012", en <http: / / www.wto.org/english/res_e/ statis_e/its2012_e/its2012_e.pdf>.

2011a "International Trade Statistics, 2011", en <http:/ / www.wto.org/spanish/res_s/ statis_s/its2011_s/its11_appendix_s.htm>.

2011b “International Trade Statitics 2011", en <http:/ / www.wto.org/english/res_e/ statis_e/its2011_e/its11_toc_e.htm>

2011c "Trade Profiles: Estados Unidos", en <http:// stat.wto.org/CountryProfile/ wSDBCountryPFView.aspx?Language $=\mathrm{E} \&$ Country $=\mathrm{CA}, \mathrm{MX}, \mathrm{CN}, \mathrm{US}>$.

2001a "International Trade Statistics 2001", en <https: / / www.wto.org/english/res_e/ statis_e/its2001_e/its01_bysector_e.htm>.

2001b "International Trade Statitics 2001", en <http: / / www.wto.org/english/res_e/ statis_e/its2001_e/stats2001_e.pdf >. 